\title{
Anatomic Success of Scleral Buckling for Rhegmatogenous Retinal Detachment - A Retrospective Study of 524 Cases
}

\author{
Christos Haritoglou ${ }^{\mathrm{a}} \quad$ Ulrich Brandlhuber ${ }^{\mathrm{a}} \quad$ Anselm Kampik $^{\mathrm{a}}$ \\ Siegfried G. Priglinger ${ }^{b}$ \\ a Department of Ophthalmology, Ludwig-Maximilians University, Munich, Germany; ${ }^{b}$ Department of \\ Ophthalmology, Linz General Hospital, Linz, Austria
}

\section{Key Words}

Scleral buckling $\cdot$ Retinal detachment $\cdot$ Anatomic success $\cdot$

Lens status

\begin{abstract}
Background/Aim: Our purpose was to investigate the anatomic success of scleral buckling surgery for rhegmatogenous retinal detachment. Material and Methods: A total of 524 consecutive patients were retrospectively analysed. Several parameters including the lens status, number of breaks and extent of retinal detachment, preoperative proliferative vitreoretinopathy and refractive errors were examined. The minimum follow-up was 6 months. The primary success rate was defined as anatomic success being stable over a period of at least 6 months after surgery. The secondary success rate was defined as anatomic success after the second intervention if necessary. Besides an analysis over all patients, the patients were grouped according to the severity of the preoperative situation in simple, medium and severe cases. $\boldsymbol{R} \boldsymbol{e}$ sults: The overall primary anatomic success rate was $84.7 \%$ and the secondary success rate $96.4 \%$ after 1 initial scleral buckling surgery and 1 additional surgery in case of persisting retinal detachment, and $19.1 \%$ of the patients with an
\end{abstract}

initially attached retina after 1 scleral buckling surgery experienced a redetachment in the postoperative course and were successfully treated in 60/85 cases. In phakic patients ( $n=359$ ) the primary success rate was $89.7 \%$, whereas in pseudophakic patients $(n=165)$ a primary success rate of 73.9\% was obtained. The primary success was additionally influenced by the extent of the retinal detachment measured in clock hours $(p<0.001)$, undetected holes $(p=0.004)$, small ( $p=0.037)$ and no gas tamponade $(p=0.021)$. In simple, medium and severe cases, phakic patients always achieved better anatomic results $(89.9,89.1$ and $90.2 \%)$ compared to pseudophakic ones (82.5, 70.3 and 36.4\%). Conclusion: Scleral buckling is a very good surgical option in phakic patients irrespective of the preoperative severity and simple cases in pseudophakic patients. Scleral buckling represents a surgical technique worth being trained and performed in the light of favourable results especially in phakic eyes.

Copyright $\odot 2010$ S. Karger AG, Basel

The data are part of U.B.'s thesis and were presented at the Club Jules Gonin 2008, Sankt Moritz, Switzerland.

\section{KARGER}

Fax +41613061234 E-Mail karger@karger.ch www.karger.com
(C) 2010 S. Karger AG, Basel

$0030-3755 / 10 / 2245-0312 \$ 26.00 / 0$

Accessible online at:

www.karger.com/oph
Christos Haritoglou, MD

Department of Ophthalmology, Ludwig-Maximilians University

Mathildenstrasse 8, DE-80336 Munich (Germany)

Tel. +49 895160 3811, Fax +498951605160

E-Mail christos.haritoglou@med.uni-muenchen.de 


\section{Introduction}

Retinal detachment represents a sight-threatening disease. The history of 'ab externo' surgery for retinal detachment was initiated by the description of the 'ignipuncture' by Jules Gonin [1], describing the drainage of subretinal fluid through a scleral incision, restoring the contact between the neurosensory retina and the underlying retinal pigment epithelium. This was followed by the concept of scleral buckling (SB) by Custodis, using elastic material to indent the sclera of the eye and thereby achieve a tamponade of the causative retinal break [2]. This procedure was further refined by Lincoff et al. by introducing silicone sponge explants and cryotherapy, and Schepens contributed the encircling band as well as indirect binocular ophthalmoscopy along with scleral depression, which revolutionized the localisation of peripheral retinal pathologies $[3,4]$.

All explant surgical techniques have in common that they allow for an effective support and treatment of the retinal pathology, which results in a reattachment of the retina in many cases. Therefore, SB surgery has been the treatment of choice for many retinal surgeons in patients with rhegmatogenous retinal detachment over the last decades. However, the technique of SB did not undergo any further significant refinement during the last 30 years.

With the invention of pars plana vitrectomy by Machemer and Parel in 1970, a second ab interno approach to the treatment of retinal detachment became available [5]. Latest developments in the field of vitreoretinal surgery imply sutureless and transconjunctival sclerotomies, aligned by an ongoing miniaturisation of the used surgical instruments [6, 7]. Nevertheless, in clinical practice the choice of the surgical approach according to the preoperative situation is not very well defined. While many surgeons may choose a scleral buckle for 'simple' retinal detachments and primary vitrectomy for 'complex' cases, there is an ongoing discussion among ophthalmic surgeons concerning which technique to apply in cases that do not fit in one of these 2 categories. As a consequence, the choice of the surgical technique is mainly based on individual preferences and experience.

In the light of new developments of transconjunctival/ transscleral vitreoretinal surgical techniques as described above, the choice of a surgical procedure for the repair of a rhegmatogenous retinal detachment is currently reappraised.

The present study was performed to evaluate the anatomic success of SB procedures performed by 2 equally trained surgeons using the same surgical technique (C.H. and S.P.) and to identify potential predictive factors influencing the surgical outcome.

\section{Material and Methods}

This is a retrospective, noncomparative study on a consecutive series of 524 patients who underwent SB surgery for the repair of rhegmatogenous retinal detachment. Preoperatively, all patients were seen by a senior surgeon and the indication for an SB procedure (or other surgical techniques) was made based on his or her clinical judgement. Patients were included in this analysis independently of the preoperative visual acuity, extent of the retinal detachment, number of retinal breaks, age, sex or lens status. For inclusion in this analysis a minimum follow-up of 6 months was required. All patients gave their written informed consent prior to surgery.

Both surgeons (C.H., S.P.) were trained by one of the authors (A.K.) and had assisted in approximately 100 surgical procedures before performing SB surgeries on their own. As a consequence, both surgeons used the same surgical technique.

The following pre- and postoperative patient data were collected from patient charts, surgical protocols and pre- and postoperative fundus drawings.

\section{Preoperative Data}

We documented the following parameters: date of birth, right or left eye, refraction (spherical equivalent), duration of symptoms (group 1: up to 3 days; group 2: 4-5 days; group 3: 6-8 days; group 4: 9-12 days; group 5: $>12$ days), lens status (phakic, pseudophakic, aphakic), macular involvement, extent of retinal detachment in clock hours, bullous or flat retinal detachment, number of retinal breaks, type and localisation of retinal breaks and preoperative proliferative vitreoretinopathy (PVR) according to Machemer et al. [8].

For analysis the patients were grouped into the following categories according to the 'severity' of the initial preoperative situation. These groups were formed on the basis of previous reports [9-11].

- Simple cases presenting with a single hole and small retinal detachment (up to 4 clock hours), \pm PVR grade A, myopia $\leq 7$ dpt.

- Medium severe cases with no or multiple holes visible, \pm PVR grade A, myopia $\leq 7 \mathrm{dpt}$.

- Severe cases with no or multiple holes visible, PVR grade B or C, myopia $>7 \mathrm{dpt}$.

\section{Intraoperative Data and Surgical Procedure}

Both surgeons applied the same operative technique. The following type and size of explant was used according to the surgeon's preference: silicone explant 2.0 or $2.5 \mathrm{~mm}$ diameter, bisected or nonbisected sponge $5 \mathrm{~mm}$ diameter, $4 \mathrm{~mm}$ encircling band and combinations. The application of an intraocular tamponade (SF6, air or BSS plus) as well as the drainage of subretinal fluid using electrolysis or a 27-gauge needle was optional. Cryopexy was always performed. Potential complications including subretinal or vitreal haemorrhage, retinal incarceration and scleral perforation during suture placement were documented as well 


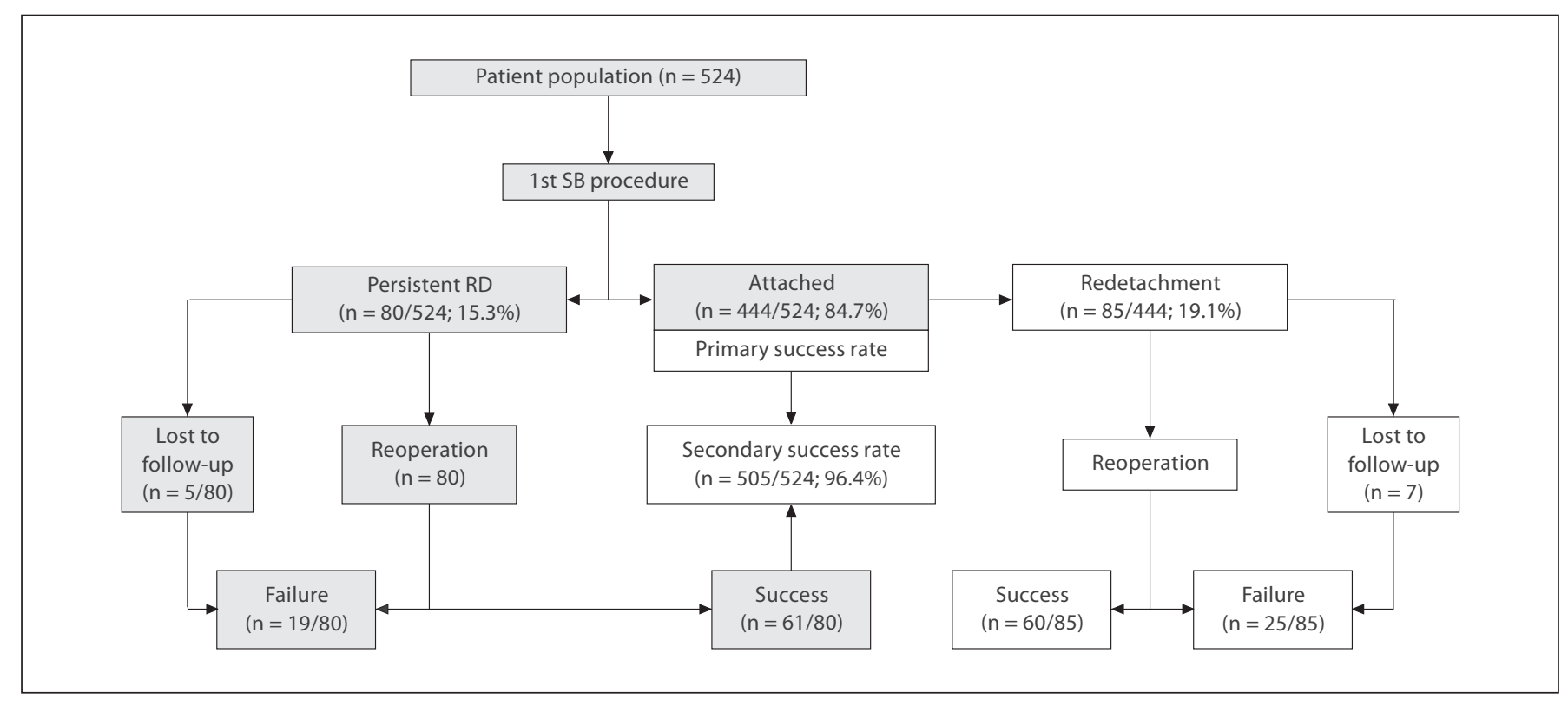

Fig. 1. Primary and secondary anatomic success after 1 initial buckle surgery and 1 subsequent surgical intervention if necessary. Patients who were lost to follow-up were considered as surgical failures and included in the respective group; 60/85 patients with redetachments in the course of follow-up were treated successfully. RD = Retinal detachment.

as the duration of surgery in minutes, the size of the buckle in relation to the extent of the retinal detachment and the number of breaks seen during surgery.

\section{Postoperative Data}

The primary success rate was defined as the number of patients presenting with a stable postoperative situation without or with minimal subretinal fluid on the day of discharge. The secondary success rate additionally included the patients treated successfully for a persistent retinal detachment. The type of surgery in cases of persistent retinal detachments and redetachments (additional buckle or buckle replacement, pneumopexy, 20-gauge vitrectomy) was chosen by the respective surgeon. Postoperative complications including diplopia, choroidal detachment, epiretinal membrane formation and endophthalmitis were documented. Patients who were lost to follow-up after a second intervention or who refused to undergo a second operation were included as 'failures' in the statistical analysis.

Visual acuity values were not further analysed because of variable examiners during the follow-up of this retrospective analysis. Data analysis was performed using SPSS 15.0. The following tests were applied: $\chi^{2}$ test (Pearson, Fisher exact test), $t$ test.

\section{Results}

\section{Results over All Patients}

We included 524 consecutive eyes of 524 patients in this analysis; $60.7 \%$ were male and $39.3 \%$ were female with a mean age of 61 years (range $=15-96$ ). The median follow-up was 16.6 months (minimum $=6$, maximum $=$ 29).

In $6.5 \%$ of the patients no break was preoperatively detectable. The number of breaks varied from 1 break in $49.4 \%, 2$ breaks in $21.4 \%, 3$ breaks in $11.3 \%$ and $>3$ breaks in $11.5 \%$. In $43.5 \%$ of the cases the retinal detachment involved up to 4 clock hours, in $47.2 \%$ 5-6 clock hours and in 9.3\% 9-12 clock hours. In $49.2 \%$ of the cases no PVR was seen prior to surgery, whereas $36.3 \%$ presented with PVR stage A, 3.1\% with PVR stage B and 11.5\% with PVR stage $\mathrm{C}$. When the initial SB surgery was performed, $68.5 \%$ of the patients were phakic and $31.5 \%$ were pseudophakic or aphakic.

The overall anatomic success rate after $1 \mathrm{SB}$ alone (primary success rate) was $84.7 \%$. In cases with persisting retinal detachments (15.3\%) a successful second procedure was performed in 61/80 eyes. We achieved an anatomic success rate of $96.4 \%$ after 1 initial SB and 1 additional surgery in case of persisting retinal detachment (secondary success rate) (fig. 1). After 1 SB, 19.1\% (85/444) of the patients with an initially attached retina experienced a redetachment (recurrent detachment) in the postoperative course and were successfully treated in 60/85 cases. Most redetachments occurred $4-8$ weeks postoperatively and 


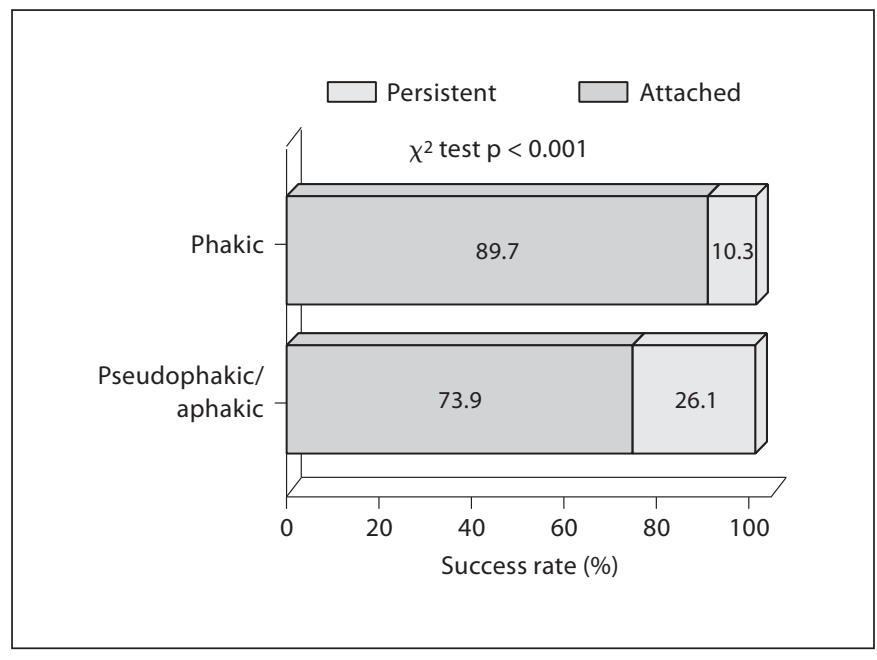

Fig. 2. Primary success rate over all patients. Patients were grouped according to the lens status in a phakic and pseudophakic/aphakic group.

Table 1. Type of interventions in patients with persisting retinal detachments or redetachments during follow-up

\begin{tabular}{|c|c|c|}
\hline & $\mathrm{n}(\%)$ & $\begin{array}{l}\text { Anatomic } \\
\text { success rate }\end{array}$ \\
\hline \multicolumn{3}{|c|}{ Surgery for persisting retinal detachment: $n=75(100 \%)$} \\
\hline Pars plana vitrectomy & $55(73.3)$ & $50(90.9)$ \\
\hline Pneumopexy & $13(17.3)$ & $7(53.8)$ \\
\hline Additional buckle/replacement & $7(9.3)$ & $4(57.1)$ \\
\hline \multicolumn{3}{|c|}{ Surgery for retinal redetachment: $n=78(100 \%)$} \\
\hline Pars plana vitrectomy & $56(71.8)$ & $44(78.6)$ \\
\hline Pneumopexy & $7(9.0)$ & $5(71.4)$ \\
\hline Additional buckle/replacement & $15(19.2)$ & $11(73.3)$ \\
\hline
\end{tabular}

were related to insufficient tamponade of the initial break (2.6\%), new breaks (41.6\%), development of PVR in the area of the buckle (35.1\%), PVR not related to the buckle (9.1\%) or unknown causes (11.7\%). The types and success rates of secondary surgical interventions in patients with persistent retinal detachments as well as redetachments are given in table 1. Patients who refused to undergo a second procedure or who were lost to follow-up were counted as 'failures' in the statistical analysis (fig. 1).

The overall primary success rate $(n=524)$ was slightly better in myopic patients with $>4 \mathrm{dpt}$. While preoperative PVR did not influence the primary success rate of the first SB procedure, a significantly higher redetachment rate was seen in patients with preoperative PVR stage C $(\mathrm{p}=0.005)$. In phakic patients $(\mathrm{n}=359)$ the primary success rate was $89.7 \%$. In pseudophakic patients $(n=165)$ we achieved a primary success rate of $73.9 \%$ (fig. 2). In addition to the lens status, the primary success of an SB procedure was negatively influenced by the extent of the retinal detachment measured in clock hours $(\mathrm{p}<0.001)$, bullous detachment $(\mathrm{p}=0.05)$, undetected holes $(\mathrm{p}=$ $0.004)$ and no gas tamponade $(\mathrm{p}=0.021)$. The size of the buckle in relation to the extent of the retinal detachment in clock hours did not significantly influence the surgical success: For buckles parallel to the limbus, the primary success rates were $78.7 \%$ in cases with the buckle being smaller than the retinal detachment, $86.7 \%$ in cases with the buckle matching the extent of the detachment and $82.2 \%$ in cases where the buckle was larger than the retinal detachment.

\section{Results according to Severity and Lens Status}

In simple cases (single hole and small retinal detachment, \pm PVR grade A, myopia $\leq 7$ diopters, $n=259$ ) the primary success rate, defined as anatomic success after the first SB procedure, was $87.6 \%$ calculated over all patients. When comparing the primary success rate in phakic $(n=179)$ and pseudophakic $(n=80)$ patients, we noted a primary success rate of $89.9 \%$ in phakic and $82.5 \%$ in pseudophakic patients ( $\mathrm{p}=0.093$; fig. 3 ). In medium severe cases (no or multiple holes visible, \pm PVR grade A, myopia $\leq 7 \mathrm{dpt} ; \mathrm{n}=203$ ) the primary success rate calculated over all patients was $82.3 \%$. With respect to the lens status of the patients included in this group, the primary success rate was $89.1 \%$ in phakic eyes $(n=129)$ and $70.3 \%$ in pseudophakic ones $(n=74 ; p<0.001$; fig. 4). Looking at the group of severe cases (no or multiple holes visible, PVR grade $\mathrm{B}$ or $\mathrm{C}$, myopia $>7 \mathrm{dpt} ; \mathrm{n}=62$ ) the overall primary success rate was $80.6 \%$. With respect to the lens status of the patients included in this group, the primary success rate was $90.2 \%$ in phakic eyes $(n=51)$ and $36.4 \%$ in pseudophakic eyes $(n=11 ; p<0.001)$ (fig. 5).

\section{Complications}

Over all 524 patients we observed the following complications: 24 patients (4.6\%) with subretinal haemorrhages not involving the macula, $2(0.4 \%)$ subretinal haemorrhages involving the macula and 21 (4\%) vitreal haemorrhages. In 3 cases $(0.6 \%)$ the retina was affected during puncture to drain subretinal fluid. We did not observe any cases of endophthalmitis, glaucoma, anterior segment ischaemia or infections of the buckle during follow-up. 


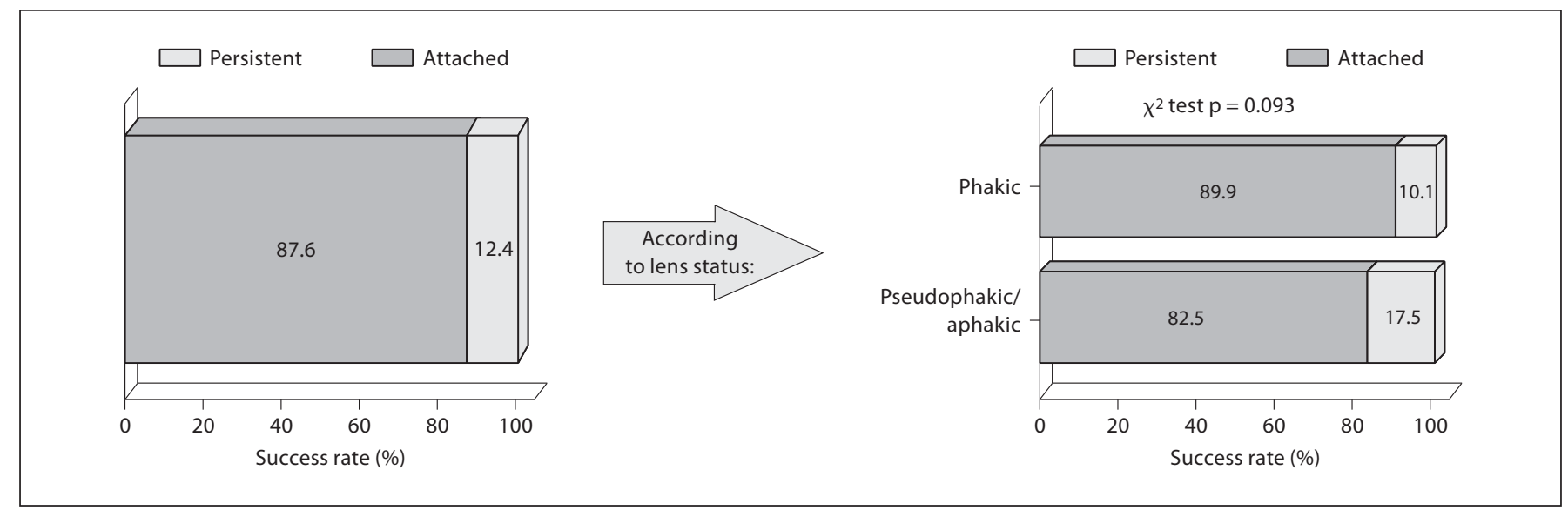

Fig. 3. Primary success rate in patients with simple retinal detachments. There is no significant difference between phakic and pseudophakic/aphakic patients.

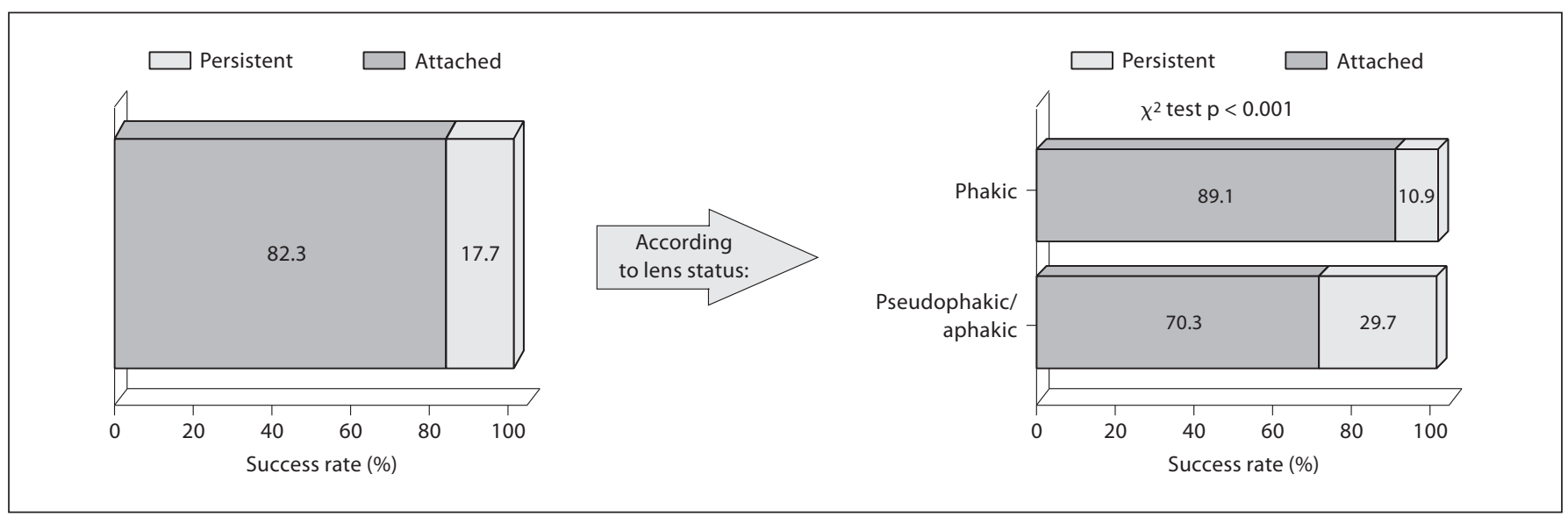

Fig. 4. Primary success rate in patients with medium severe retinal detachments. Comparing phakic and pseudophakic/aphakic patients, there was a significant difference concerning the anatomic outcome $(\mathrm{p}<0.001)$.

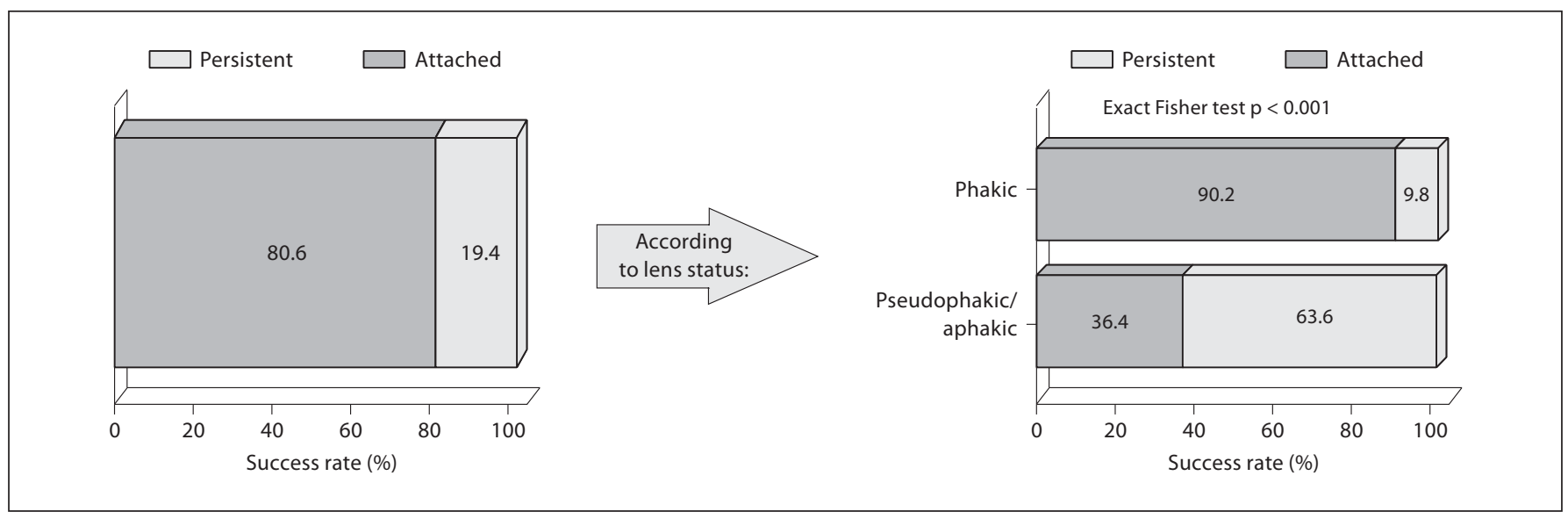

Fig. 5. Primary success rate in patients with severe retinal detachments. The anatomic success rate in pseudophakic patients is only $36.4 \%$ ( $\mathrm{p}<0.001)$. 


\section{Discussion}

Our findings indicate that SB surgery results in a good anatomic outcome in a large patient collective. The overall primary success rate is within the range reported in the literature [12-15]. The lens status of the patient appears to be very relevant as a main predictive factor for surgical success. While good primary anatomic success rates of approximately $90 \%$ could be obtained in phakic patients irrespective of the severity of the retinal detachment, less favourable results were seen in pseudophakic patients in simple, medium and severe cases, with drastically decreasing success rates according to the severity of the preoperative situation. In addition, redetachments in the postoperative course following an SB procedure are higher in pseudophakic patients compared to phakic ones.

In general, the success rates, both anatomic and functional, of different surgical approaches to treat retinal detachments are difficult to analyse as they may be influenced by a large number of factors. This includes the number of breaks, the extent of the retinal detachment, the presence of PVR before surgery, the involvement of the macular region, the height of the retinal detachment (bullous or not), refractive errors, the lens status and others. The effect of these factors on the functional and anatomic outcome of retinal detachment surgery has been previously investigated in the 'Scleral Buckling versus Primary Vitrectomy in Rhegmatogenous Retinal Detachment (SPR)' trials [9-11], which were designed to compare SB surgery and primary pars plana vitrectomy in rhegmatogenous retinal detachments of medium complexity. The categories of severity described in our study are based on the inclusion and exclusion criteria of the SPR trail [11]. In contrast to the SPR study, which included only cases of medium severity, we additionally included cases which were categorized as simple and severe. Therefore, only the anatomic results obtained for medium complex cases in our study may be compared to the results seen in the SPR trials. The SPR study reported a primary anatomic success rate of $63.6 \%$ in phakic patients and $53.4 \%$ in pseudophakic patients, compared to 89.1 and $70.3 \%$ in our investigation. The redetachment rates were also different with $26.3 \%$ (phakic eyes) versus $39.9 \%$ (pseudophakic eyes) in the SPR study and 13.0 versus $19.2 \%$ in ours. Although the SPR trial and our own study should be compared with care due to different study designs, these differences may be explained by the large number of surgeons participating in the SPR trial and the resulting heterogenous surgical techniques used by each surgeon. However, as seen in the SPR trial, we were able to demonstrate that scleral buckle procedures are more successful in phakic eyes compared to pseudophakic eyes in cases of medium complexity with respect to the primary anatomic success and the rate of redetachment.

We are of course aware of certain limitations of the present study which are related to the retrospective design of this investigation and the lack of a control group. As a consequence, we did not analyse functional results of our patients, as they had been seen by different examiners when presenting for follow-up visits. The strength of the present study is the large number of patients included and the fact that all patients were operated on by 2 equally experienced surgeons using the same surgical technique. Despite its limitations, our investigation represents a 'real-life setting' in a single-centre analysis.

Based on our findings we believe that SB may still be considered an appropriate approach to simple and medium severe cases of rhegmatogenous retinal detachments especially for phakic eyes and simple cases in pseudophakic eyes until a superiority of primary vitrectomy for these cases has been proven. In addition, it appears worthwhile to save a clear lens by avoiding primary vitrectomy in cases without PVR or with advanced severity. Therefore, appropriate training of SB surgery for rhegmatogenous retinal detachments is still necessary and justified in view of the favourable results especially in phakic patients.

\section{References}

1 Gonin J: Pathogénie et anatomie pathologique des décollements rétiniens. Bull Mem Soc Ophthalmol 1920;33:1.

2 Hoerauf H, Heimann $\mathrm{H}$, Hansen L, Laqua $\mathrm{H}$ : Scleral buckling surgery and pneumatic retinopexy: techniques, indications and results. Ophthalmologe 2008;105:7-18.
3 Michels RG, Wilkinson CP, Rice TA: History of retinal detachment surgery; in Retinal Detachment. St. Louis, Mosby, 1990, pp 243 323.

4 Lincoff $\mathrm{H}$, Baras I, et al: Modification of the Custodis procedure for retinal detachment. Arch Ophthalmol 1965;173:160-163.
5 Machemer R, Buettner H, Norton EW, Parel JM: Vitrectomy: a pars plana approach. Trans Am Acad Ophthalmol Otolaryngol 1971;75:813-820.

6 Tsang CW, Cheung BT, Lam RF, Lee GK, Yuen CY, Lai TY, Lam DS: Primary 23-gauge transconjunctival sutureless vitrectomy for rhegmatogenous retinal detachment. Retina 2008;28:1075-1081. 
7 Bartz-Schmidt U, Szurman P, Wong D, Kirchhof B: New developments in retinal detachment surgery. Ophthalmologe 2008;105: 27-36.

8 Machemer R, Aaberg TM, Freeman HM, Irvine AR, Lean JS, Michels RM: An updated classification of retinal detachment with proliferative vitreoretinopathy. Am J Ophthalmol 1991;112:159-165.

9 Heimann H, Hellmich M, Bornfeld N, BartzSchmidt KU, Hilgers RD, Foerster MH: Scleral buckling versus primary vitrectomy in rhegmatogenous retinal detachment (SPR Study): design issues and implications. SPR Study report No 1. Graefes Arch Clin Exp Ophthalmol 2001;239:567-574.
10 Feltgen N, Weiss C, Wolf S, Ottenberg D, Heimann H; SPR Study Group: Scleral buckling versus primary vitrectomy in rhegmatogenous retinal detachment study (SPR Study): recruitment list evaluation. Study report No 2. Graefes Arch Clin Exp Ophthalmol 2007;245:803-809.

11 Heimann H, Bartz-Schmidt KU, Bornfeld N Weiss C, Hilgers RD, Foerster MH, Scleral Buckling versus Primary Vitrectomy in Rhegmatogenous Retinal Detachment Study Group: Scleral buckling versus primary vitrectomy in rhegmatogenous retinal detachment: a prospective randomized multicenter clinical study. Ophthalmology 2007;114: 2142-2154.

12 Mahdizadeh M, Masoumpour M, Ashraf H: Anatomical retinal reattachment after scleral buckling with and without retinopexy: a pilot study. Acta Ophthalmol 2008;86:297301.
13 Falkner-Radler CI, Binder S: Long-term results of scleral buckling using a microsurgical approach. Klin Monatsbl Augenheilkd 2008;225:1055-1061.

14 Brazitikos PD, Androudi S, Christen WG, Stangos NT: Primary pars plana vitrectomy versus scleral buckle surgery for the treatment of pseudophakic retinal detachment: a randomized clinical trial. Retina 2005;25: 957-964.

15 Figueroa MS, Corte MD, Sbordone S, Romano A, Alvarez MT, Villalba SJ, Schirru A: Scleral buckling technique without retinopexy for treatment of rhegmatogeneous retinal detachment: a pilot study. Retina 2002; $22: 288-293$. 